\title{
Diosmetin reduces bone loss and osteoclastogenesis by regulating the expression of TRPV1 in osteoporosis rats
}

\author{
Song Hu ${ }^{1 \#}$, Youyi Huang ${ }^{2 *}$, Yong Chen ${ }^{1}$, Renyi Zhou ${ }^{3}$, Xiaozhong Yang ${ }^{1}$, Yi Zou ${ }^{1}$, Daxin Gao ${ }^{1}$, \\ Hua Huang ${ }^{1}$, Dongming $\mathbf{Y u}^{1}$ \\ ${ }^{1}$ Department of Orthopedics, West China-Guang'an Hospital, Sichuan University, Guang'an, China; ${ }^{2}$ Medical Department of Nanchang University, \\ Nanchang, China; ${ }^{3}$ Department of Orthopedics, First hospital of China Medical University, Shenyang, China \\ Contributions: (I) Conception and design: S Hu, Y Huang, D Gao; (II) Administrative support: Y Chen, R Zhou, D Gao; (III) Provision of study \\ materials or patients: S Hu, Y Huang, X Yang, Y Zou; (IV) Collection and assembly of data: S Hu, Y Huang, H Huang, D Yu; (V) Data analysis and \\ interpretation: S Hu, Y Huang, Y Chen, R Zhou, X Yang, Y Zou, H Huang; (VI) Manuscript writing: All authors; (VII) Final approval of manuscript: \\ All authors. \\ \#These authors contributed equally to this work. \\ Correspondence to: Daxin Gao. Department of Orthopedics, West China-Guang'an Hospital, Sichuan University, No. 1, Section 4, Binhe Road, \\ Guang'an District, Guang'an 638001, China. Email: DaxinGao123@163.com.
}

Background: Osteoporosis is a systemic skeletal disorder and occurs frequently in postmenopausal women and older men. This study aimed to examine whether diosmetin (DIO) can relieve estrogen deficiencyinduced osteoporosis and to explore the underlying mechanisms of this potential effect.

Methods: Forty-nine Sprague-Dawley (SD) rats were divided into seven groups. Six groups underwent bilateral ovariectomy (OVX), while the sham group underwent ovarian exposure surgery. DIO and evodiamine were administered 3 days before surgery, and then subcutaneously every 3 days for 3 months in the following fashion: group I, DIO (100 mg/kg); group II, OVX; group III, OVX + DIO (50 mg/kg); group IV, OVX + DIO (100 mg/kg); group V, OVX + evodiamine (10 mg/kg) group; group VI, OVX + DIO $(100 \mathrm{mg} / \mathrm{kg})+$ evodiamine $(10 \mathrm{mg} / \mathrm{kg})$ group. Bone histopathological damage, bone loss, osteoclast production, and the expression level of transient receptor potential vanilloid 1 (TRPV1) were detected.

Results: Compared with the sham group, the expression of bone resorption-related genes, osteoclastassociated receptor (OSCAR) $\left(1.00 \% \pm 0.16 \%\right.$ versus $\left.4.5 \% \pm 0.28 \%,{ }^{* *}, \mathrm{P}<0.01\right)$ and tartrate-resistant acid phosphatase (TRAP) $\left(2.0 \% \pm 0.6 \%\right.$ versus $\left.18.00 \pm 1.2 \%,{ }^{* * *}, \mathrm{P}<0.001\right)$, was increased significantly. The protein level of osteogenic marker proteins, osterix (Osx) $(1.0 \% \pm 0.1 \%$ versus $0.03 \% \pm 0.01 \%, * *, \mathrm{P}<0.01)$ and type 1 collagen $(\mathrm{COL} 1 \mathrm{~A} 1)\left(1.0 \% \pm 0.13 \%\right.$ versus $\left.0.13 \% \pm 0.05 \%,{ }^{* *}, \mathrm{P}<0.01\right)$ was decreased significantly with the increase of TRPV1 $\left(1.0 \% \pm 0.15 \%\right.$ versus $\left.2.89 \% \pm 0.28 \%,{ }^{* *}, \mathrm{P}<0.01\right)$ protein level. Notably, DIO can alleviate some abnormal symptoms related to osteoporosis.

Conclusions: DIO can relieve typical osteoporosis symptoms in an OVX osteoporosis rat model. The underlying mechanism may be associated with the downregulation of TRPV1.

Keywords: Osteoporosis; diosmetin (DIO); transient receptor potential vanilloid 1 (TRPV1); bone loss; osteoclastogenesis; reduction

Submitted Aug 17, 2020. Accepted for publication Oct 19, 2020.

doi: $10.21037 /$ atm-20-6309

View this article at: http://dx.doi.org/10.21037/atm-20-6309 


\section{Introduction}

Osteoporosis is a systemic skeletal disorder that can occur in a variety of bones throughout the body and is characterized by low bone mineral density (BMD), abnormal microstructure of bone tissue, increased bone fragility, and a high risk of fracture (1). It is well known that the main form of bone metabolism is bone remodeling. Bone remodeling is mainly performed via osteoblasts and osteoclasts. Osteoclasts absorb and promote the degradation of old bone while osteoblasts synthesize new bones and maintain the source of the new bone. The delicate balance between bone resorption and bone formation is essential for maintaining healthy bone mass and preventing osteoporosis (2-4). Currently, the drugs used for postmenopausal osteoporosis are bisphosphonates, selective estrogen receptor modulators, calcitonin, estrogen, recombinant human parathyroid hormone fragments, and others. Higher adverse reactions, such as typical femoral fractures, bisphosphonate-related osteonecrosis of the jaw, hypercalcemia, thromboembolism, vaginal bleeding, and even breast cancer and endometrial cancer, severely limit the widespread use of these drugs. Hence, drugs with satisfactory efficacy and small adverse reactions are worth studying and developing.

Transient receptor potential vanilloid 1 (TRPV1) is a ligand-gated nonselective cation channel, which can be activated by capsaicin and other stimuli such as low $\mathrm{pH}$ and noxious heat (5). Early studies of TRPV1 focused on inflammatory nociception $(6,7)$, while a recent report has shown that TRPV1 is associated with bone pain (8). In a mouse model, TRPV1 antagonists were shown to significantly alleviate bone pain (9), while numbers of TRPV1-ir neurons were also found to dramatically increase in an osteoporosis femur rat model (10). TRPV1 is emerging as a focus in osteoporosis research, which has now turned towards developing TRPV1 agonists/desensitizers (11). According to reports, TRPV1 channel is expressed in bone cells and plays a critical role in the metabolism of these bone cells (12), which indicates that TRPV1 channel may be a promising therapeutic strategy for treating osteoporosis pain.

Evodiamine, a naturally occurring indole alkaloid, is one of the main bioactive ingredients of Evodiae fructus (13). Evidence suggests that evodiamine can decrease oxidative stress and suppresses inflammatory cytokine expression (14). Evodiamine has also been recognized as a multi-target drug because it has evolved a prominent ability to bind various proteins (13). Yang et al. reported that evodiamine can act as an TRPV1 agonist in the reversion of tumor-associated fibroblast (TAF)-induced epithelial-mesenchymal transition (EMT) in normal colonic epithelial cells (15). In the present study, evodiamine was used as a TRPV1 agonist to study and confirm the inhibitory mechanism of diosmetin (DIO) on osteoporosis.

Flavonoids are a class of phenolic compounds commonly found in medicinal plants, fruit, and vegetables. Flavonoids have been shown to be involved in a variety of biological functions, including the induction of osteoblast differentiation (16-18). DIO is a natural flavonoid found in acacia and olive leaves and can induce a variety of pharmacological effects, including those of anticancer, antibacterial, antioxidant, and anti-inflammatory activity (19). Studies have also found that DIO has the ability to promote the differentiation and maturation of osteoblasts (20), but there is a lack of research on DIO in the relief of osteoporosis.

The main purpose of this study was to investigate whether DIO can exert remission effects on bone loss and osteoclastogenesis in estrogen deficiency-induced osteoporosis and to explore the underlying mechanisms of DIO regulation on TRPV1 expression in an osteoporosis rat model. We present the following article in accordance with the ARRIVE reporting checklist (available at http:// dx.doi.org/10.21037/atm-20-6309).

\section{Methods}

\section{Reagents}

DIO (purity $\geq 98 \%$ ) was purchased from Shanghai Jianglai Biotechnology Co., Ltd., while evodiamine (purity $\geq 99 \%$ ) was purchased from Hepeng (Shanghai) Biotechnology Co., Ltd. A TRAP Staining Kit was purchased from Beijing Shengke Boyuan Biotechnology Co., Ltd, and a Bicinchoninic Acid Kit was purchased from Shanghai Yanxi Biotechnology Co., Ltd. RIPA buffer was purchased from Shanghai Biyuntian Biotechnology Co. Ltd and a polymerase chain reaction (PCR) kit was purchased from Shanghai Xiya Biotechnology Co., Ltd.

\section{Animals}

Experimental animals were purchased from the Chengdu University of Traditional Chinese Medicine (Chengdu, Sichuan, China). Sprague-Dawley (SD) rats were housed 
in a 12 -h light-dark cycle in a $21-23{ }^{\circ} \mathrm{C}$ environment and allowed free access to food and water.

All animal experiments were approved by West ChinaGuang'an Hospital, Sichuan University (No. HXGA2019074) and conducted according to the declaration of the NIH Guide for the Care and Use of Laboratory Animals.

\section{Osteoporosis model}

Forty-nine 8-week-old, female SD rats were cultured for 1 week under controlled conditions to adapt the environment and were then divided into seven groups. Six groups underwent bilateral ovariectomy (OVX), while the sham group underwent only ovarian exposure surgery. DIO and evodiamine were administered 3 days before surgery, and then subcutaneously every 3 days for 3 months in the following fashion: group I, DIO $(100 \mathrm{mg} / \mathrm{kg})$; group II, OVX; group III, OVX + DIO $(50 \mathrm{mg} / \mathrm{kg})$; group IV, OVX + DIO (100 mg/kg); group V, OVX + evodiamine (10 mg/kg); group VI, OVX + DIO (100 mg/kg) + evodiamine $(10 \mathrm{mg} / \mathrm{kg})$ group. The dose of DIO was determined with reference to previous experimental studies and slightly modified (20). After 12 weeks of treatment, the animals were then euthanized, blood samples were taken from the right common carotid artery of the rats, and the femur was removed for histopathological examination.

Evodiamine, an effective agonist of TRPV1, was used in this study. The dose of evodiamine was in accordance with that used in a previous study $(21,22)$.

\section{Histopatbology}

For histomorphometric analysis, the right femur was stored in a $10 \%$ formaldehyde fixative for $24 \mathrm{~h}$ at room temperature. The sample was placed in $10 \%$ formic acid for 28 days to decalcify and then routinely examined using an optical microscope. The decalcified fixed left femur was cross-sectioned into $3 \mu \mathrm{m}$ thick sections using microtome. Hematoxylin and eosin (HE) staining was used to observe femoral injury under light microscopy of five randomly selected fields per slice.

\section{Bone mineral density (BMD) determination}

According to a previous study, experimental animals underwent dual-energy $\mathrm{X}$-ray absorptiometry. The BMD of the proximal third of the right tibia was detected at a scan speed of $60 \mathrm{~mm} / \mathrm{s}$ and a scan pitch of $1.5 \mathrm{~mm}$. The whole femur, including the distal femur bone and the femoral shaft, was divided into three equal segments for X-ray analysis.

\section{Osteocalcin and alkaline phosphatase levels determination}

Collected blood $(2 \mathrm{~mL})$ was centrifuged at 2,000 $\times \mathrm{g}$ for $15 \mathrm{~min}$ at $4{ }^{\circ} \mathrm{C}$. The osteocalcin and alkaline phosphatase (ALP) serum levels were detected by measuring the absorbance at $450 \mathrm{~nm}$ (23).

\section{Bone morphometric parameters determination}

Trabecula number (Tb.N), bone volume over total volume (BV/TV), and trabecula separation (Tb.Sp) were acquired by analyzing volume of interest (VOI) (24). The operator performing the scan analysis turned a was blinded to the treatment method associated with the specimen.

\section{Tartrate-resistant acid phosphatase (TRAP) staining}

The production of osteoclasts in bone homogenate was detected by TRAP staining (25). Bone homogenate was fixed with $100 \%$ methanol. Fixed samples were stored in $\mathrm{L}$ (+)-tartrate buffer $(0.335 \mathrm{~mol} / \mathrm{L}, \mathrm{pH} 4.9 \pm 0.1)$ and incubated with a TRAP kit for $5 \mathrm{~min}$ at $37^{\circ} \mathrm{C}$. Upon staining, TRAPstained areas were examined by an optical microscope. Multinucleated osteoclasts with three or more nuclei were confirmed as TRAP-positive cells. TRAP activity was quantified by optical density analysis using Image J software. All experiments were performed in triplicate with independent samples.

\section{PCR}

Expression of osteogenic marker proteins osterix (Osx), type 1 collagen (COL1A1), distal-less homeobox 2 (DLX2), and osteoclast-associated receptor (OSCAR) and TRAP in bone homogenate were detected by PCR. After different treatment, total RNA was isolated from bone homogenate using Trizol Reagent. Then, the kits (Xiya Biotechnology, Shanghai, China) were used to strictly measure the levels of Osx, COL1A1, DLX2, OSCAR and TRAP according to the kit's instructions.

\section{Western blot analysis}

Protein from femurs was isolated with RIPA buffer and 


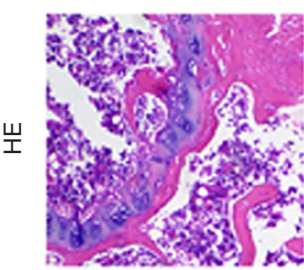

Sham

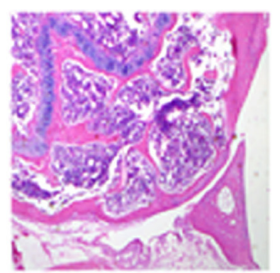

DIO $(1000 \mathrm{mg} / \mathrm{kg})$

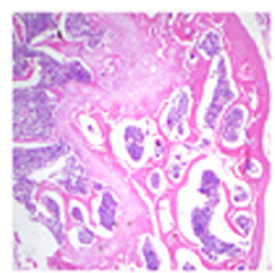

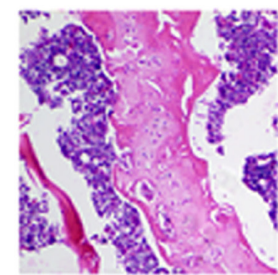

DIO $(50 \mathrm{mg} / \mathrm{kg})$

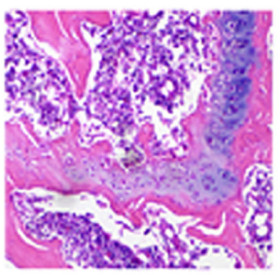

DIO (100 mg/kg)

OVX

Figure 1 DIO mitigates bone histopathological damage. Bone histopathological damage was detected by HE staining. Representative pictures of $\mathrm{HE}$ (magnification $\times 200$ ). The results are presented as mean $\pm \mathrm{SD}$ and represent three individual experiments. Grouping situation ( $\mathrm{n}=8)$ : the sham group; the DIO (100 mg/kg) group; the OVX group; the OVX + DIO (50 mg/kg) group; the OVX + DIO $(100 \mathrm{mg} / \mathrm{kg})$ group. DIO, diosmetin; HE, hematoxylin and eosin; OVX, ovariectomy.

was quantified with a bicinchoninic acid kit. Total protein samples $(20 \mu \mathrm{g})$ were loaded into $10 \%$ sodium dodecyl sulfate polyacrylamide gel electrophoresis (SDS-PAGE) loading buffer and were subsequently transferred to polyvinylidene difluoride (PVDF) membranes. The PVDF membranes were sealed with $5 \%$ skimmed milk at $37^{\circ} \mathrm{C}$ for $120 \mathrm{~min}$. Next, they were incubated overnight at $4{ }^{\circ} \mathrm{C}$ with the following primary antibodies: anti-Osx (ab94744, 1:1,000), antiCOL1A1 (ab34710, 1:1,000), anti-DLX2 (ab152341, 1:1,000) and TRPV1 (ab159807, 1:1,000). Then, PVDF membranes and horseradish peroxidase (HRP) were incubated at $37{ }^{\circ} \mathrm{C}$ for $60 \mathrm{~min}$. Band densities were determined and analyzed with the Bio-Rad CFX-96 automatic digital gel image analysis system (Bio-Rad, CA, USA).

\section{Statistical analysis}

The statistical analysis was conducted with SPSS 21.0 (SPSS Inc., Chicago, IL, USA). Data are presented as mean \pm standard deviation (SD). Multiple comparisons of parametric data were measured by one-way analysis of variance (ANOVA). A $\mathrm{P}$ value $<0.05$ was considered statistically significant.

\section{Results}

\section{DIO mitigated histopathological bone damage}

The bone cortex around the femur of the sham group was thick and dense; meanwhile, the size of the bone marrow cavity was moderate, the size, thickness, and arrangement of the cortical bone were normal, the bone cell size was normal, and the bone cell was located in the bone lacuna.
The bone cells were closely embedded in the wall of the bone sag and had no holes (Figure 1).

The structure of the femur in the DIO group was not significantly different from that in the sham group. In the OVX group, the cortical bone around the femur became thinner, the trabecular bone of the femur became smaller and shorter, and the gap between the trabecular bones was significantly larger than that of the other three groups. Atrophy of bone cells became smaller, and the gap between bone cells and the bone wall was significantly increased, indicating osteoporosis. Compared with the OVX group, the OVX group + DIO $(50 \mathrm{mg} / \mathrm{kg} /$ day $)$ group and the OVX group + DIO $(100 \mathrm{mg} / \mathrm{kg} /$ day $)$ had thicker cortical bone around the femur and a more uniform density of trabecular bone. The interstitial trabecular bone space was small, the bone cell size was normal, and there was no obvious cavity between the bone cells and the bone lacuna. These results suggest that DIO treatment significantly improved the osteoporosis condition.

\section{DIO reduced bone loss}

There was no significant difference in BMD, Tb.N, Tb.Sp, $\mathrm{BV} / \mathrm{TV}, \mathrm{ALP}$ activity, or osteocalcin content between the DIO group and the sham group (Figure 2).

In the OVX group, BMD, Tb.N, and BV/TV were significantly reduced. Upon DIO treatment, these abnormalities were improved, and the improvement was increased in a concentration-dependent manner. In contrast, Tb.Sp, ALP activity, and osteocalcin levels were significantly increased, and DIO treatment significantly reduced the abnormal increase of the above three indicators, 
A

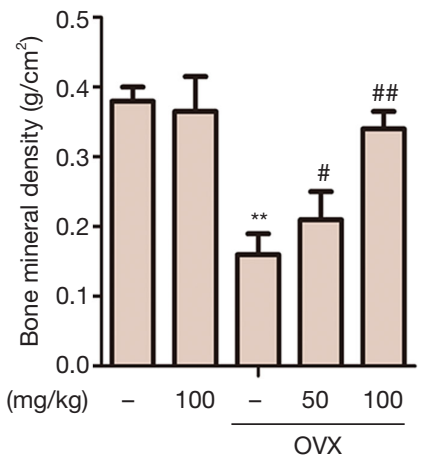

C

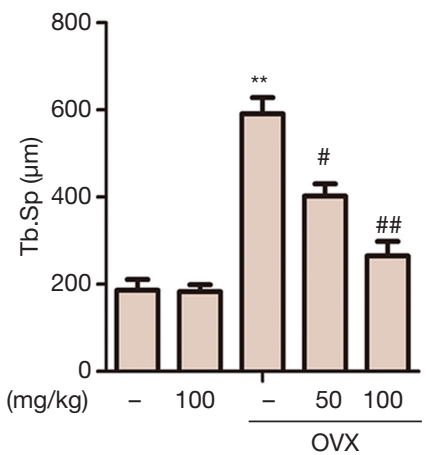

$E$

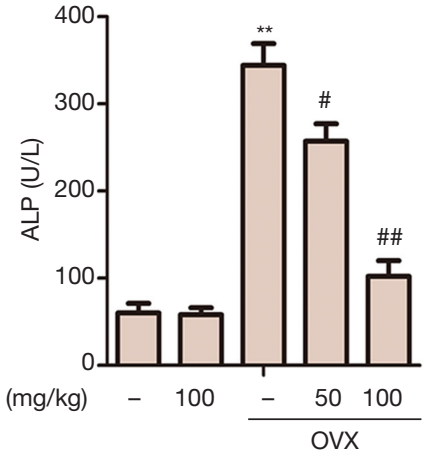

B
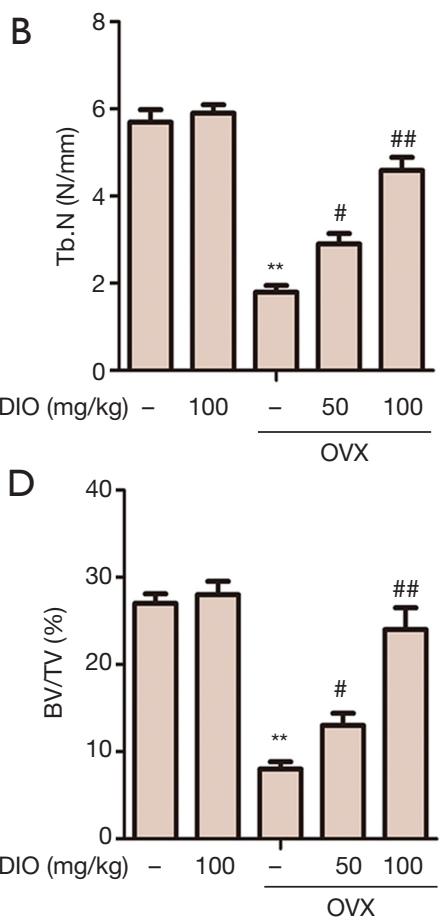

$\mathrm{F}$

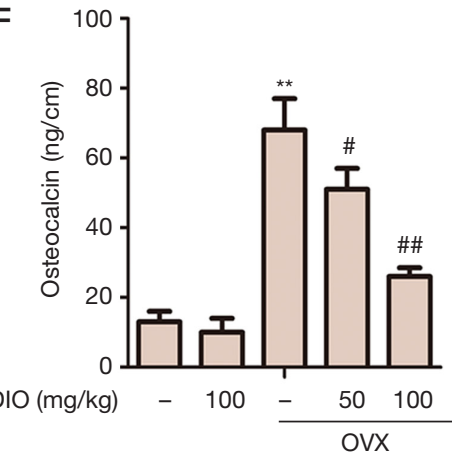

Figure 2 DIO reduces bone loss. Bone loss was evaluated by six markers. (A) Bone mineral density $\left(\mathrm{g} / \mathrm{cm}^{2}\right)$; (B) Tb.N (N/mm); (C) Tb.Sp $(\mu \mathrm{m}) ;(\mathrm{D}) \mathrm{BV} / \mathrm{TV}(\%)$; (E) ALP $(\mathrm{U} / \mathrm{L}) ;(\mathrm{F})$ osteocalcin $(\mathrm{ng} / \mathrm{cm})$. The results are presented as mean $\pm \mathrm{SD}$ and represent three individual experiments. ${ }^{* *}, \mathrm{P}<0.01$, versus the sham group; ${ }^{\#}, \mathrm{P}<0.05,{ }^{\#}, \mathrm{P}<0.01$ versus the OVX group. DIO, diosmetin; BV/TV, bone volume over total volume; ALP, alkaline phosphatase; OVX, ovariectomy.

with the effect appearing in a concentration-dependent manner. The results indicated that DIO treatment could effectively reduce bone loss.

\section{DIO inbibited the production of osteoclasts}

TRAP staining was used to detect the production of osteoclasts. The number of TRAP-positive cells was significantly increased in the OVX group compared to the sham group, and DIO treatment effectively attenuated this abnormal increase (Figure $3 A$ ). To further explore the inhibition of DIO on osteoclastogenesis, PCR was used to detect the expression of bone resorption-related genes, OSCAR and TRAP, in bone homogenates. As shown in Figure 3B,C, compared with the sham group, the relative expression level of OSCAR and TRAP increased significantly in the OVX group, and DIO treatment effectively alleviated this abnormal increase. Therefore, DIO was found to inhibit the production of osteoclasts. 

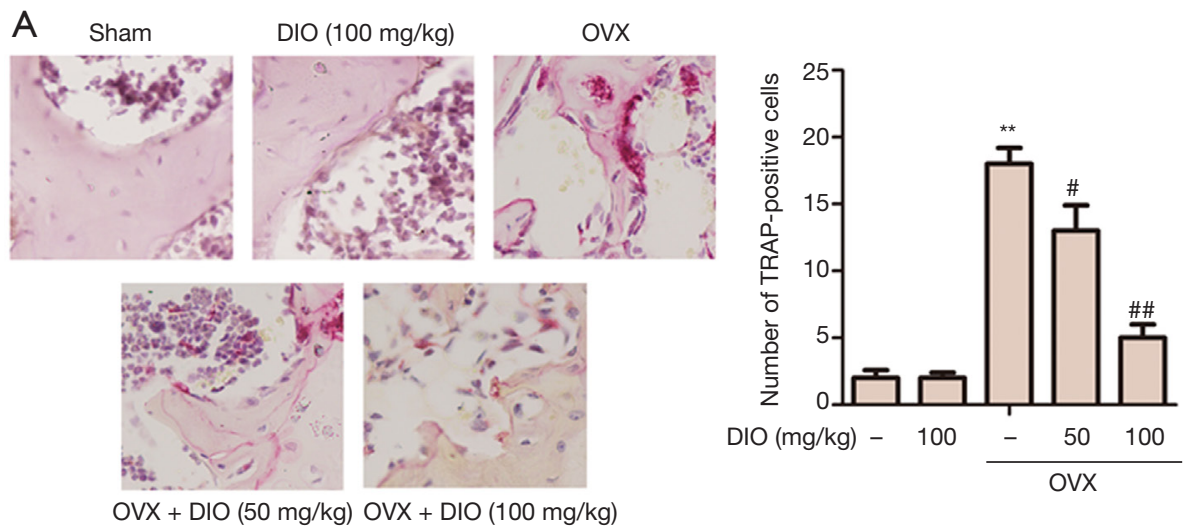

B

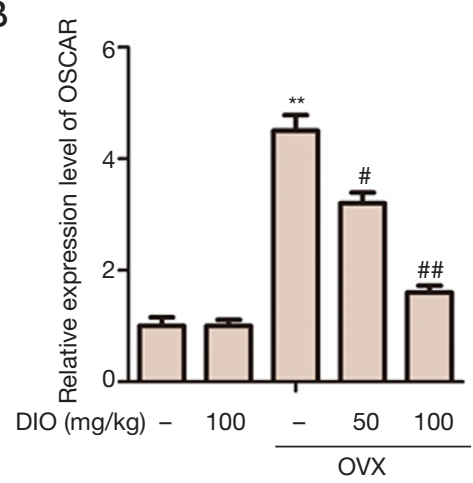

C

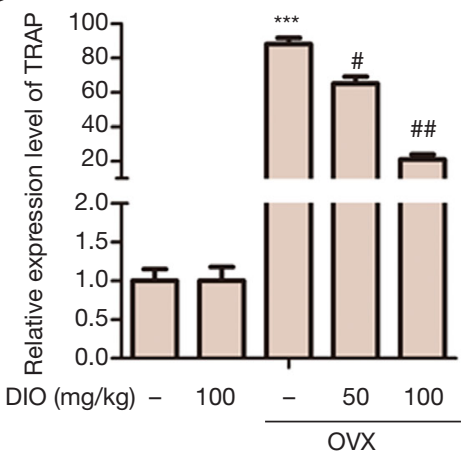

Figure 3 DIO inhibits the production of osteoclasts. The production of osteoclasts was detected by TRAP staining. (A) Representative pictures of TRAP staining and number of TRAP-positive cells (magnification $\times 200$ ); the expression of bone resorption-related genes, OSCAR and TRAP, in bone homogenate was detected by PCR. Relative expression level of OSCAR (B) and TRAP (C). The results are presented as mean $\pm \mathrm{SD}$ and represent three individual experiments. ${ }^{* *}, \mathrm{P}<0.01$, and ${ }^{* * *}, \mathrm{P}<0.001$ versus the sham group; ${ }^{*} \mathrm{P}<0.05$ and ${ }^{\# \#}$, $\mathrm{P}<0.01$ versus the OVX group. DIO, diosmetin; TRAP, tartrate-resistant acid phosphatase; OSCAR, osteoclast-associated receptor; PCR, polymerase chain reaction; OVX, ovariectomy.

\section{DIO promoted the expression of osteogenic marker proteins}

Relative mRNA expression levels of Osx, COL1A1 and DLX2 were detected by PCR. There was no significant difference in the relative mRNA expression levels of Osx, COL1A1, or DLX2 in the sham group and the DIO group (Figure $4 A, B, C$ ). The relative mRNA expression levels of Osx, COL1A1, and DLX2 in the OVX group were significantly lower than those in the sham group. DIO treatments had significantly improved these anomalies in a concentration-dependent manner. The relative protein expression levels of Osx, COL1A1, and DLX2 were examined by western blotting. The relative protein expression levels of Osx, COL1A1, and DLX2 in each group were similar to those of mRNA expression levels, which indicated that DIO could effectively promote the expression of osteogenic marker proteins, Osx, COL1A1, and DLX2.

\section{DIO reduced bone loss and osteoclast production by downregulating the expression of TRPV1}

The protein expression level of TRPV1 was lower in the sham group and DIO group, while it was upregulated significantly in the OVX group (Figure 5A). DIO treatment attenuated the abnormal expression of TRPV1 protein level in a dose-dependent manner. After the addition of the TRPV1 agonist evodiamine $(10 \mathrm{mg} / \mathrm{kg})$, the protein expression level of TRPV1 was significantly upregulated in the OVX + evodiamine $(10 \mathrm{mg} / \mathrm{kg})$ group (Figure $5 B)$. After the TRPV1 agonist evodiamine $(10 \mathrm{mg} / \mathrm{kg})$ was added, the BMD, Tb.N, and BV/TV in the OVX + DIO 
A

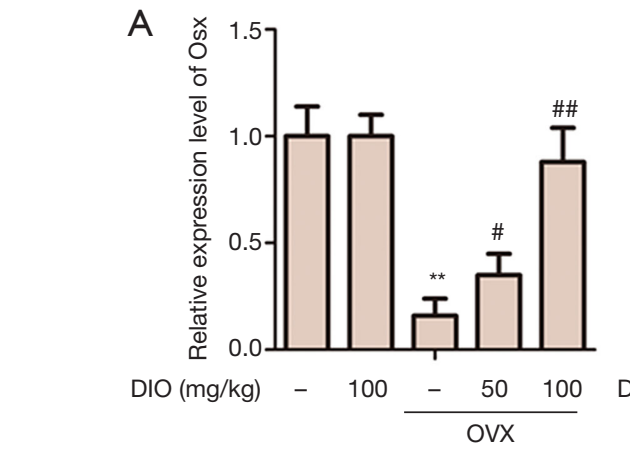

D

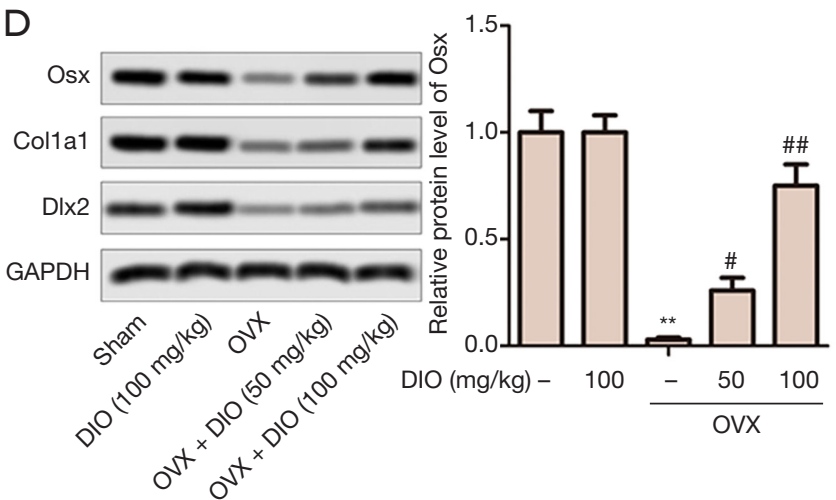

B

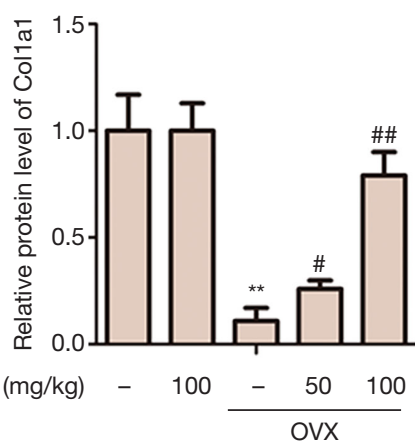

C

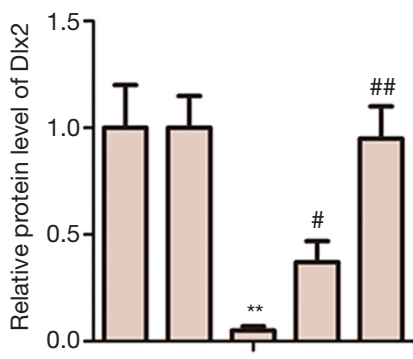

$\mathrm{DIO}(\mathrm{mg} / \mathrm{kg}) \quad-100 \quad-50 \quad 100$
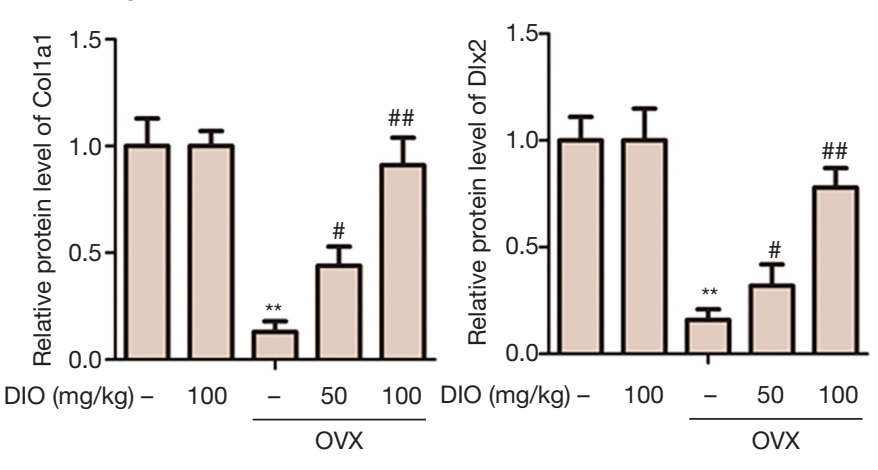

Figure 4 DIO promotes the expression of osteogenic marker proteins. The miRNA level expression of Osx, COL1A1, and DLX2 was detected by PCR. The relative expression level of Osx (A), COL1A1 (B), and DLX2 (C). The protein level expression of Osx, COL1A1, and DLX2 was detected by western blotting. (D) Representative pictures of western blotting and the relative expression level of Osx, COL1A1, and DLX2. The results are presented as mean $\pm \mathrm{SD}$ and represent three individual experiments. ${ }^{* *}, \mathrm{P}<0.01$, versus the sham group; ${ }^{*}, \mathrm{P}<0.05$ and $^{\# \#}, \mathrm{P}<0.01$ versus the OVX group. DIO, diosmetin; PCR, polymerase chain reaction; OVX, ovariectomy.

$(100 \mathrm{mg} / \mathrm{kg} /$ day $)+$ evodiamine $(10 \mathrm{mg} / \mathrm{kg})$ group were significantly elevated compared to those in the OVX + evodiamine $(10 \mathrm{mg} / \mathrm{kg})$ group, , and this was accompanied by a significant decrease of Tb.Sp content (Figure 5C,D,E,F). Compared with the OVX group, the number of TRAP-positive cells in the OVX + evodiamine $(10 \mathrm{mg} / \mathrm{kg}$ ) group was obviously upregulated (Figure $5 G)$. Compared with the OVX + evodiamine $(10 \mathrm{mg} / \mathrm{kg})$ group, the number of TRAP-positive cells in the OVX + DIO $(100 \mathrm{mg} / \mathrm{kg} /$ day $)+$ evodiamine $(10 \mathrm{mg} / \mathrm{kg})$ group was significantly reduced.

The expression of osteogenic marker proteins Osx, COL1A1, and DLX2 in bone homogenate was detected by western blotting. As shown in Figure 5 H, the protein expression levels of Osx, COL1A1, and DLX2 were significantly downregulated in the OVX + evodiamine $(10 \mathrm{mg} / \mathrm{kg})$ group compared with the OVX group, and significantly upregulated in the OVX group + DIO $(100 \mathrm{mg} / \mathrm{kg} / \mathrm{day})+$ evodiamine $(10 \mathrm{mg} / \mathrm{kg})$ group. The above results indicated that DIO could reduce bone loss and osteoclast production by downregulating the expression of TRPV1.

\section{Discussion}

Osteoporosis includes both primary and secondary osteoporosis. Postmenopausal osteoporosis, which belongs to primary osteoporosis, is the most common type of osteoporosis. This is one of the reasons why the incidence of osteoporosis in women is higher than that of men $(26,27)$. Postmenopausal osteoporosis is caused by estrogen deficiency, which leads to increased bone turnover, and ultimately to a loss of connectivity in the trabecular bone and cortical pores of cortical bone $(28,29)$. In the present study, an ovariectomized rat model was established to simulate postmenopausal osteoporosis. The results of our study revealed that DIO dramatically mitigated bone histopathological damage, reduced bone loss, inhibited the 

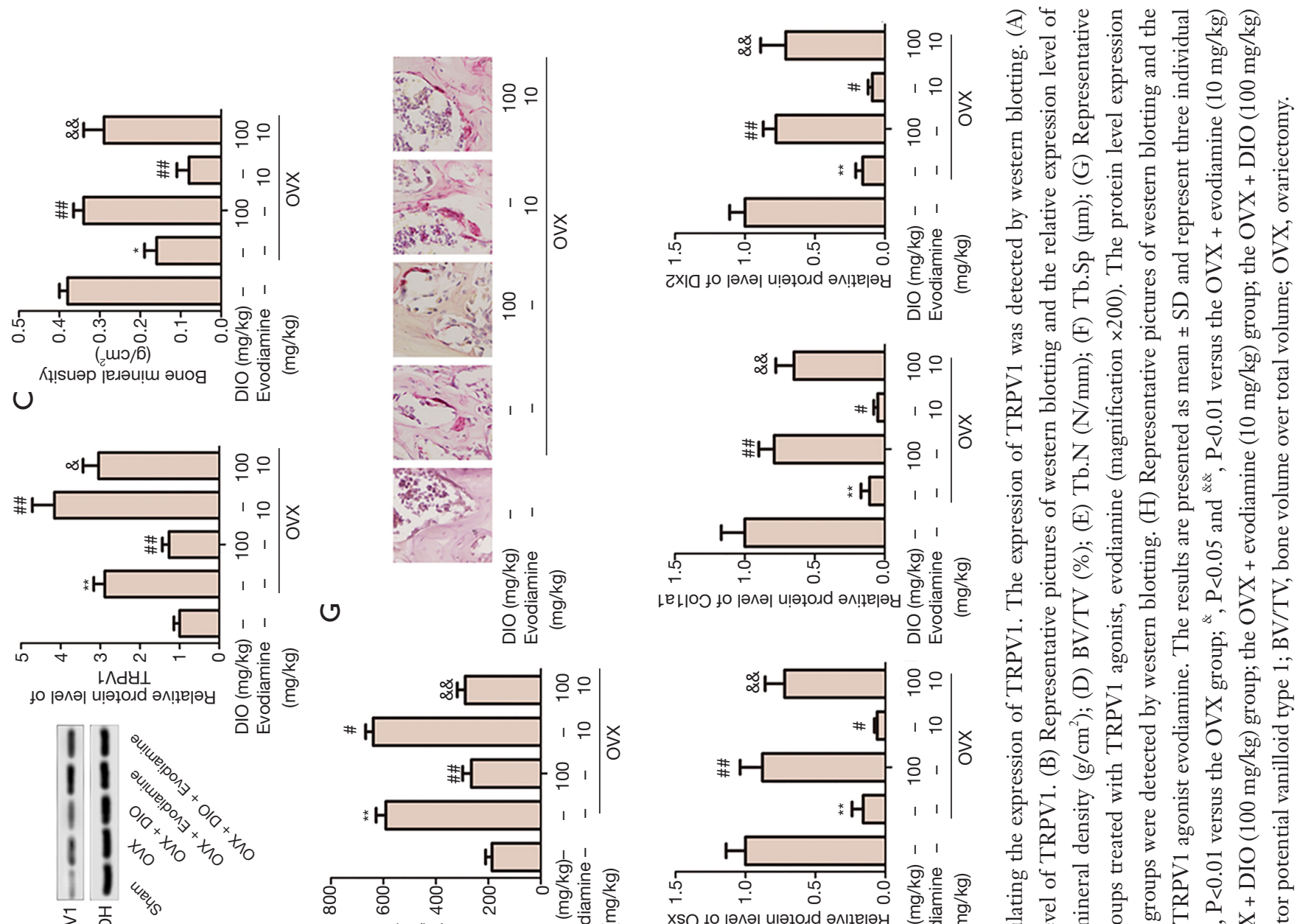

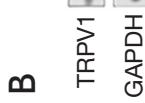

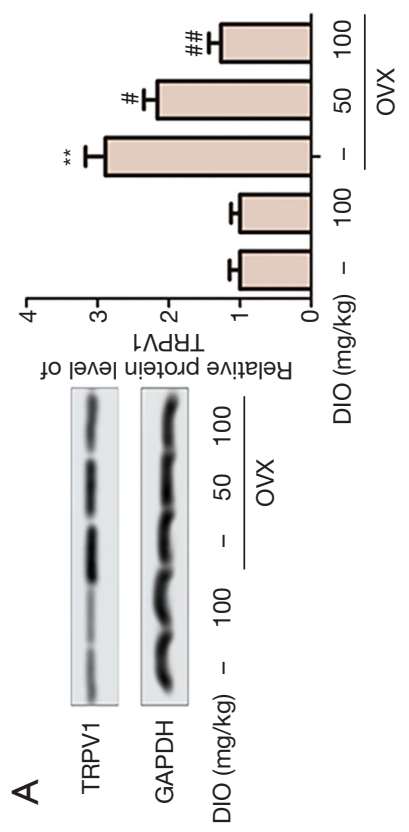

ᄂ

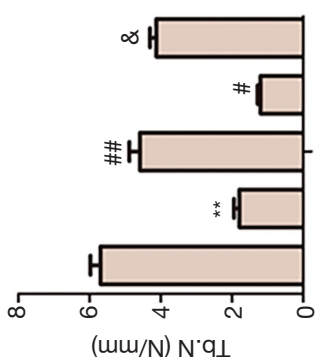

ш

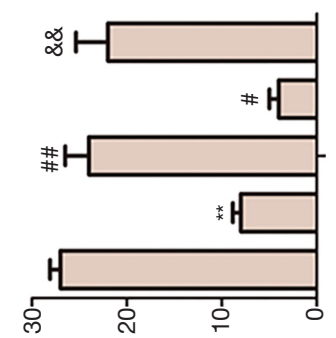

(\%) $\Lambda$ ก/
음
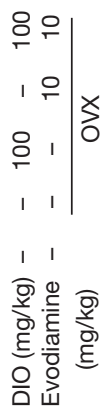

I

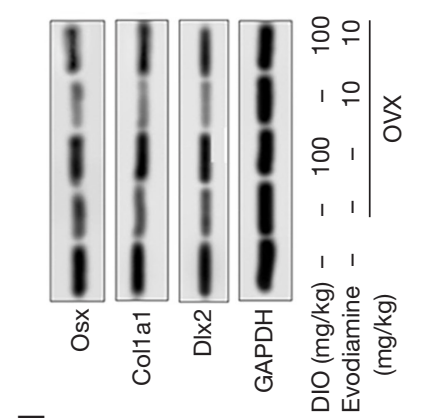

우울
1
1

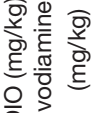

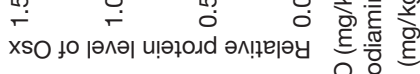
믐

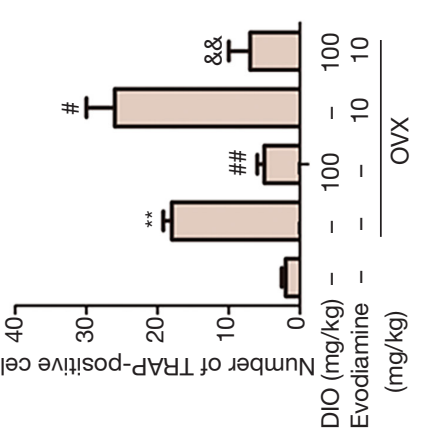

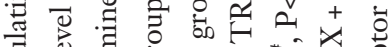

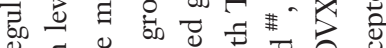

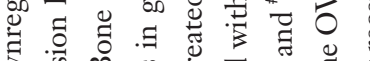

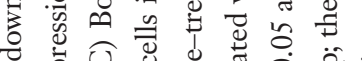

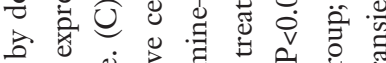

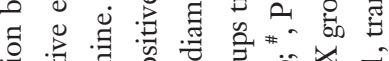

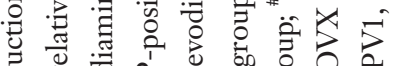
च ग्ञ

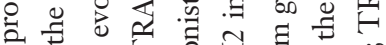

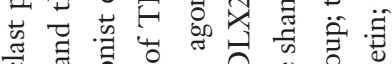
ర大

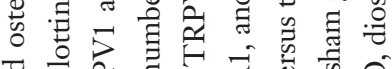

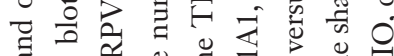

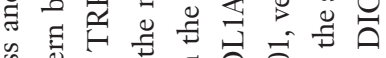

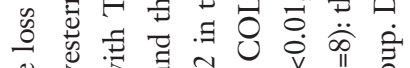

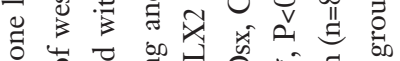
章

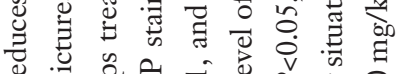

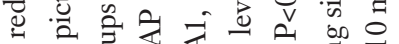

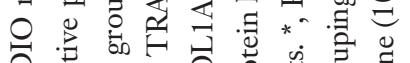

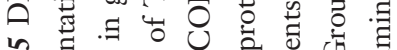
in 50

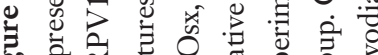

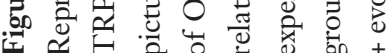


production of osteoclasts, and promoted the expression of osteogenic marker proteins. Simultaneously, we found that the expression of TRPV1 was upregulated in the osteoporosis rat model. Notably, compared with the addition of TRPV1 agonist evodiamine, we found that DIO reduced bone loss and osteoclast production by downregulating the expression of TRPV1. Thus, DIO administration could be a promising therapeutic strategy for postmenopausal osteoporosis.

DIO is a natural flavonoid that is derived from citrus fruit and has been identified in analgesic Amphilophium crucigerum (L.). Studies on DIO have typically focused on its anticancer, antioxidant, and anti-inflammatory properties (19,30). In recent years, however, a considerable number of studies have reported that DIO is involved in skeletal system repair. The literature indicates that DIO can be an effective therapy in osteolytic bone diseases by suppressing osteoclast formation and activation (31). This is consistent with our finding that DIO could reduce bone loss and inhibit the production of osteoclasts. Bhattacharyya et al. (32) demonstrated DIO to be an selective estrogen receptor- $\beta$ agonist that inhibits the anti-osteoblastogenic Wnt antagonist and stimulates osteoblast differentiation. Hsu and Kuo (20) reported that DIO has the ability to promote the differentiation and maturation of osteoblasts. These results are in line with the observations derived in the present study, which indicated that DIO could promote the expression of osteogenic marker proteins Osx, COL1A1, and DLX2. Importantly, DIO was shown to possess a positive skeletal effect in the regulation of dynamic bone balance and may be a potential drug in osteoporosis treatment, thus warranting further study. Indeed, the molecular mechanisms underlying the pharmacological benefits of DIO for osteoporosis have not been comprehensively elucidated.

TRPV1 is considered to be a cAMP-activated protein (CAP) receptor and exhibits significant gating diversity in response to numerous physical and chemical stimuli. In recent years, TRPV1 has attracted attention as it was found to mediate the occurrence of bone disease. Yoshino et al. (10) reported that TRPV1 may play a part in osteoporosis femur sensory perception. Under normal physiological conditions, TRPV1 is contained in dorsal root ganglion (DRG) neurons that innervate the femur. TRPV1 expression can reflect the number of TRPV1-ir neurons in the DRG-dominant osteoporosis femur in rats. Meanwhile, another study found that OVX in mice resulted in mechanical hyperalgesia and osteoporosis, and upregulated
TRPV1 channel expression in DRG neurons (33). In line with these findings, we found that the protein expression level of TRPV1 was significantly increased in the OVX osteoporosis rat model. Pan et al. (34) reported that TRPV1channel-related $\mathrm{Ca}^{2+}$ plays an important role in osteoblasts in cytokinesis induced by singlenucleotide polymorphisms (SNPs). Adamante et al. (35) identified DIO as a TRPV1 antagonist in different mice pain models.

In this study, evodiamine significantly enhanced the protein expression level of TRPV1, and decreased expression levels of osteogenic marker proteins, Osx, COL1A1, and DLX2. Fortunately, DIO treatment reversed these adverse symptoms. In addition, the TRPV1 agonist evodiamine control experiment indicated that the addition of DIO significantly decreased the bone markers BMD, Tb.N, Tb.Sp, and BV/TV. In parallel, DIO dramatically decreased the number of TRAP-positive cells in the TRAP staining assay, which suggested that DIO inhibited the production of osteoclasts. These two observations indicate that DIO reduced bone loss and inhibited the production of osteoclasts via downregulating the expression of TRPV1 in the OVX osteoporosis model rats. This conclusion was in agreement with a previous study result in which TRPV1 gene knockout decreased the number of osteoclasts, impaired the formation and absorption of osteoclasts, and diminished the frequency of oscillation and the peak concentration of cytoplasmic calcium of osteoclast precursor cells (36). Furthermore, numerous studies have shown that TRPV1 plays an excitatory role in stimulating osteoclast activity (37-39), and thus the downregulation of TPRV1 channel expression may be an effective mitigation for postmenopausal osteoporosis. Bones are dynamic organs that make up the skeletal structure of vertebrates (40).

In conclusion, the results of the present study indicate that DIO is a potential therapeutic agent for osteoporosis in an OVX rat model. We found that DIO could mitigate bone histopathological damage, reduce bone loss, inhibit the production of osteoclasts, and promote the expression of osteogenic marker proteins. The underlying mechanism for these effects involves the remission of bone loss and the reduction of osteoclastogenesis via downregulating the expression of TRPV1 channel. It should be noted, however, that the present study was implemented in animal models with a limited sample size and thus its clinical significance should not be overstated. The possibility of the offtargeting of the TRPV1 agonist, evodiamine, cannot be absolutely precluded. More in-depth studies are needed to 
verify the preventive effect of DIO on osteoporosis.

\section{Acknowledgments}

Funding: None.

\section{Footnote}

Reporting Checklist: The authors have completed the ARRIVE reporting checklist. Available at http://dx.doi. org/10.21037/atm-20-6309

Data Sharing Statement: Available at http://dx.doi. org/10.21037/atm-20-6309

Conflicts of Interest: All authors have completed the ICMJE uniform disclosure form (available at http://dx.doi. org/10.21037/atm-20-6309). The authors have no conflicts of interest to declare.

Ethical Statement: The authors are accountable for all aspects of the work in ensuring that questions related to the accuracy or integrity of any part of the work are appropriately investigated and resolved. All animal experiments were approved by West China-Guang'an Hospital, Sichuan University (No. HXGA2019074) and conducted according to the declaration of the NIH Guide for the Care and Use of Laboratory Animals.

Open Access Statement: This is an Open Access article distributed in accordance with the Creative Commons Attribution-NonCommercial-NoDerivs 4.0 International License (CC BY-NC-ND 4.0), which permits the noncommercial replication and distribution of the article with the strict proviso that no changes or edits are made and the original work is properly cited (including links to both the formal publication through the relevant DOI and the license). See: https://creativecommons.org/licenses/by-nc-nd/4.0/.

\section{References}

1. Glaser DL, Kaplan FS. Osteoporosis. Definition and clinical presentation. Spine (Phila Pa 1976) 1997;22:12s-6s.

2. Chen X, Zhang S, Chen X, et al. Emodin promotes the osteogenesis of MC3T3-E1 cells via BMP-9/Smad pathway and exerts a preventive effect in ovariectomized rats. Acta Biochim Biophys Sin (Shanghai) 2017;49:867-78.

3. Xu X, Jia X, Mo L, et al. Intestinal microbiota: a potential target for the treatment of postmenopausal osteoporosis. Bone Res 2017;5:17046.

4. Wang XR, Kwok TCY, Griffith JF, et al. Prevalence of cervical spine degenerative changes in elderly population and its weak association with aging, neck pain, and osteoporosis. Ann Transl Med 2019;7:486.

5. Tominaga M, Caterina MJ, Malmberg AB, et al. The cloned capsaicin receptor integrates multiple painproducing stimuli. Neuron 1998;21:531-43.

6. Guo A, Vulchanova L, Wang J, et al. Immunocytochemical localization of the vanilloid receptor 1 (VR1): relationship to neuropeptides, the $\mathrm{P} 2 \mathrm{X} 3$ purinoceptor and IB4 binding sites. Eur J Neurosci 1999;11:946-58.

7. Yu L, Yang F, Luo H, et al. The role of TRPV1 in different subtypes of dorsal root ganglion neurons in rat chronic inflammatory nociception induced by complete Freund's adjuvant. Mol Pain 2008;4:61.

8. Niiyama Y, Kawamata T, Yamamoto J, et al. Bone cancer increases transient receptor potential vanilloid subfamily 1 expression within distinct subpopulations of dorsal root ganglion neurons. Neuroscience 2007;148:560-72.

9. Niiyama Y, Kawamata T, Yamamoto J, et al. SB366791, a TRPV1 antagonist, potentiates analgesic effects of systemic morphine in a murine model of bone cancer pain. Br J Anaesth 2009;102:251-8.

10. Yoshino K, Suzuki M, Kawarai Y, et al. Increase of TRPV1-immunoreactivity in dorsal root ganglia neurons innervating the femur in a rat model of osteoporosis. Yonsei Med J 2014;55:1600-5.

11. Holzer P, Izzo AA. The pharmacology of TRP channels. Br J Pharmacol 2014;171:2469-73.

12. Wang J, Lu HX, Wang J. Cannabinoid receptors in osteoporosis and osteoporotic pain: a narrative update of review. J Pharm Pharmacol 2019;71:1469-74.

13. Yu H, Jin H, Gong W, et al. Pharmacological actions of multi-target-directed evodiamine. Molecules 2013;18:1826-43.

14. Yuan SM, Gao K, Wang DM, et al. Evodiamine improves congnitive abilities in SAMP8 and APP(swe)/PS1( E9) transgenic mouse models of Alzheimer's disease. Acta Pharmacol Sin 2011;32:295-302.

15. Yang $W$, Gong $X$, Wang $X$, et al. A mediator of phosphorylated Smad2/3, evodiamine, in the reversion of TAF-induced EMT in normal colonic epithelial cells. Invest New Drugs 2019;37:865-75.

16. Morris C, Thorpe J, Ambrosio L, et al. The soybean isoflavone genistein induces differentiation of MG63 human osteosarcoma osteoblasts. J Nutr 2006;136:1166-70. 
17. Choi EM. Apigenin increases osteoblastic differentiation and inhibits tumor necrosis factor-alpha-induced production of interleukin-6 and nitric oxide in osteoblastic MC3T3-E1 cells. Pharmazie 2007;62:216-20.

18. Hsu YL, Chang JK, Tsai CH, et al. Myricetin induces human osteoblast differentiation through bone morphogenetic protein-2/p38 mitogen-activated protein kinase pathway. Biochem Pharmacol 2007;73:504-14.

19. Patel K, Gadewar M, Tahilyani V, et al. A review on pharmacological and analytical aspects of diosmetin: a concise report. Chin J Integr Med 2013;19:792-800.

20. Hsu YL, Kuo PL. Diosmetin induces human osteoblastic differentiation through the protein kinase C/p38 and extracellular signal-regulated kinase $1 / 2$ pathway. J Bone Miner Res 2008;23:949-60.

21. Ogasawara M, Matsubara T, Suzuki H. Inhibitory effects of evodiamine on in vitro invasion and experimental lung metastasis of murine colon cancer cells. Biol Pharm Bull 2001;24:917-20.

22. Ko YH, Shim KY, Lee SY, et al. Evodiamine Reduces Caffeine-Induced Sleep Disturbances and Excitation in Mice. Biomol Ther (Seoul) 2018;26:432-8.

23. Wang X, Chen L, Peng W. Protective effects of resveratrol on osteoporosis via activation of the SIRT1-NF- $\kappa \mathrm{B}$ signaling pathway in rats. Exp Ther Med 2017;14:5032-8.

24. Zhang R, Liu ZG, Li C, et al. Du-Zhong (Eucommia ulmoides Oliv.) cortex extract prevent OVX-induced osteoporosis in rats. Bone 2009;45:553-9.

25. Sekiguchi Y, Mano H, Nakatani S, et al. Mangiferin positively regulates osteoblast differentiation and suppresses osteoclast differentiation. Mol Med Rep 2017;16:1328-32.

26. Sharma N, Natung T, Barooah R, et al. Effect of Multiparity and Prolonged Lactation on Bone Mineral Density. J Menopausal Med 2016;22:161-6.

27. Zhu Q, Shan C, Li L, et al. Differential expression of genes associated with hypoxia pathway on bone marrow stem cells in osteoporosis patients with different bone mass index. Ann Transl Med 2019;7:309.

28. Diab DL, Watts NB. Postmenopausal osteoporosis. Curr Opin Endocrinol Diabetes Obes 2013;20:501-9.

29. Black DM, Rosen CJ. Clinical Practice. Postmenopausal Osteoporosis. N Engl J Med 2016;374:254-62.

30. Yang Y, Gong XB, Huang LG, et al. Diosmetin exerts antioxidative, anti-inflammatory and anti-apoptotic effects to protect against endotoxin-induced acute hepatic failure in mice. Oncotarget 2017;8:30723-33.
31. Shao S, Fu F, Wang Z, et al. Diosmetin inhibits osteoclast formation and differentiation and prevents LPS-induced osteolysis in mice. J Cell Physiol 2019;234:12701-13.

32. Bhattacharyya S, Pal S, Mohamed R, et al. A nutraceutical composition containing diosmin and hesperidin has osteogenic and anti-resorptive effects and expands the anabolic window of teriparatide. Biomed Pharmacother 2019;118:109207.

33. Naito Y, Wakabayashi H, Kato S, et al. Alendronate inhibits hyperalgesia and suppresses neuropeptide markers of pain in a mouse model of osteoporosis. J Orthop Sci 2017;22:771-7.

34. Pan L, Song K, Hu F, et al. Nitric oxide induces apoptosis associated with TRPV1 channel-mediated $\mathrm{Ca}(2+)$ entry via S-nitrosylation in osteoblasts. Eur J Pharmacol 2013;715:280-5.

35. Adamante G, de Almeida AS, Rigo FK, et al. Diosmetin as a novel transient receptor potential vanilloid 1 antagonist with antinociceptive activity in mice. Life Sci 2019;216:215-26.

36. He LH, Liu M, He Y, et al. TRPV1 deletion impaired fracture healing and inhibited osteoclast and osteoblast differentiation. Sci Rep 2017;7:42385.

37. Idris AI, van 't Hof RJ, Greig IR, et al. Regulation of bone mass, bone loss and osteoclast activity by cannabinoid receptors. Nat Med 2005;11:774-9.

38. Bab I, Ofek O, Tam J, et al. Endocannabinoids and the regulation of bone metabolism. J Neuroendocrinol 2008;20 Suppl 1:69-74.

39. Idris AI, Sophocleous A, Landao-Bassonga E, et al. Cannabinoid receptor type 1 protects against age-related osteoporosis by regulating osteoblast and adipocyte differentiation in marrow stromal cells. Cell Metab 2009;10:139-47.

40. Arnett TR, Orriss IR. Metabolic properties of the osteoclast. Bone 2018;115:25-30.

(English Language Editor: J. Gray)

Cite this article as: $\mathrm{Hu} \mathrm{S}$, Huang $\mathrm{Y}$, Chen $\mathrm{Y}$, Zhou R, Yang X, Zou Y, Gao D, Huang H, Yu D. Diosmetin reduces bone loss and osteoclastogenesis by regulating the expression of TRPV1 in osteoporosis rats. Ann Transl Med 2020;8(20):1312. doi: 10.21037/atm-20-6309 